\title{
Disminución del nivel de conciencia: ¿causa abdominal?
}

\author{
E. La Orden Izquierdo ${ }^{\mathrm{a}}$, M. Fernández lbieta ${ }^{\mathrm{b}}$, I. Cuadrado Pérez ${ }^{\mathrm{b}}$, \\ C. Palomino Muñoz ${ }^{\mathrm{b}}$, JT. Ramos Amador ${ }^{\mathrm{b}}$ \\ `MIR-Pediatría. Servicio de Pediatría. Hospital Universitario de Getafe. Madrid. España. \\ ${ }^{\natural}$ Pediatra. Servicio de Pediatría. Hospital Universitario de Getafe. Madrid. España.
}

\section{Resumen}

La invaginación intestinal es una causa frecuente de abdomen agudo y obstrucción intestinal en niños de entre 3 meses y 6 años de edad. Describimos el caso clínico de un niño de 2 años que acude a urgencias por un episodio de cefalea, vómitos y escasa reactividad posterior frente a estímulos. Tras un estudio inicial para la exclusión de las causas potenciales de la alteración del nivel de conciencia, la realización de una ecografía abdominal dio el diagnóstico de invaginación ileo-ileal. La resolución posterior fue espontánea. Las causas abdominales deben ser excluidas en la edad pediátrica ante alteraciones del nivel de conciencia inexplicadas por otros cuadros. La ecografía abdominal es una técnica no invasiva que puede ayudar al diagnóstico.

Palabras clave: Invaginación intestinal. Alteración del nivel de conciencia. Dolor abdominal.

\section{Altered consciousness: abdominal origin?}

\section{Abstract}

Intussusception is a common cause of acute abdomen and intestinal obstruction in children between 3 months and 6 years old. We describe a case of a 2-year-old child who went to the emergency department for an episode of headache, vomiting and low reactivity to stimuli. After an initial study to rule out potential causes of altered mental status, an abdominal ultrasound gave the diagnosis of ileo-ileal intussusception. The subsequent resolution was spontaneous. Abdominal causes should be excluded in the pediatric age in case of changes in level of consciousness that are unexplained by other conditions. Abdominal ultrasound is a noninvasive technique that can help the diagnosis.

Key words: Intestinal invagination. Altered consciousness. Abdominal pain.

Enrique La Orden Izquierdo, e_o_izquierdo@yahoo.es

Los autores declaran no presentar conflictos de intereses en relación con la preparación y publicación de este artículo. 


\section{Introducción}

La invaginación intestinal es una causa frecuente de abdomen agudo y obstrucción intestinal en niños de entre 3 meses y 6 años de edad. Suele cursar como episodios de dolor abdominal cólico paroxístico intenso repetido en intervalos frecuentes. Síntomas como la letargia y la alteración del nivel de conciencia pueden preceder a estos síntomas. El diagnóstico de invaginación intestinal debe tenerse en cuenta en pacientes que presentan alteraciones inexplicables de conciencia sin signos de focalidad neurológica.

\section{Caso clínico}

Varón de 2 años que acude al servicio de urgencias por un episodio de cefalea y vómito junto con decaimiento e hipotonía muscular tras rechazo de una toma, sin pérdida de conocimiento, de segundos de duración, con buena recuperación posterior, y que queda pálido y sudoroso. No presenta signos de focalidad neurológica ni refiere ingesta de medicamentos. Afebril. No presenta otros síntomas asociados.

Exploración física: temperatura: $36^{\circ} \mathrm{C}$; peso: $13,6 \mathrm{~kg}$; tensión arterial: 114/60 $\mathrm{mm} / \mathrm{Hg}$; frecuencia cardíaca: $91 \mathrm{lpm}$; saturación transcutánea de oxígeno: $100 \%$ con aire ambiental; palidez muco- cutánea con buena hidratación. Resto de la exploración neurológica y por aparatos: normal, salvo por una escasa reactividad a los estímulos.

Pruebas complementarias: en el hemograma, leucocitos: 14.300 (segmentados: $69 \%$, linfocitos: $23 \%$, monocitos: $6 \%$ y eosinófilos: $2 \%$ ); hemoglobina: 10,9 $\mathrm{g} / \mathrm{dl}$; volumen corpuscular medio: 73 ; plaquetas: $412.000 / \mathrm{ml}$. Gasometría: ph: 7,37; $\mathrm{PCO}_{2}: 44$; bicarbonato: 26 . Glucosa sérica: $90 \mathrm{mg} / \mathrm{dl}$; creatinina: 0,4 mg/dl; $\mathrm{Na}$ : $139 \mathrm{mEq} / \mathrm{l}, \mathrm{K}^{+} 3,8 \mathrm{mEq} / \mathrm{l}$. Proteína C reactiva: 0,4 mg/l; transaminasa glutámico oxalacética: 33; transaminasa glutámico pirúvica: 24 . Tomografía computarizada (TC) craneal: sin alteraciones. Tóxicos en orina: negativos. Punción lumbar: 0 células.

Evolución: durante su observación en urgencias presenta 4 vómitos y tendencia al sueño; se mantiene afebril en todo momento. Se le realiza una ecografía abdominal (figuras 1, 2 y 3 ) en la que se observa una invaginación ileo-ileal y es remitido al servicio de cirugía pediátrica del hospital de referencia, donde se repite la ecografía. No se encuentran entonces signos de dicha invaginación pero sí varias adenopatías mesentéricas. El paciente permaneció en observación 24 horas y fue dado de alta sin complicaciones. 
Figura 1. Imagen ecográfica de adenopatías mesentéricas.

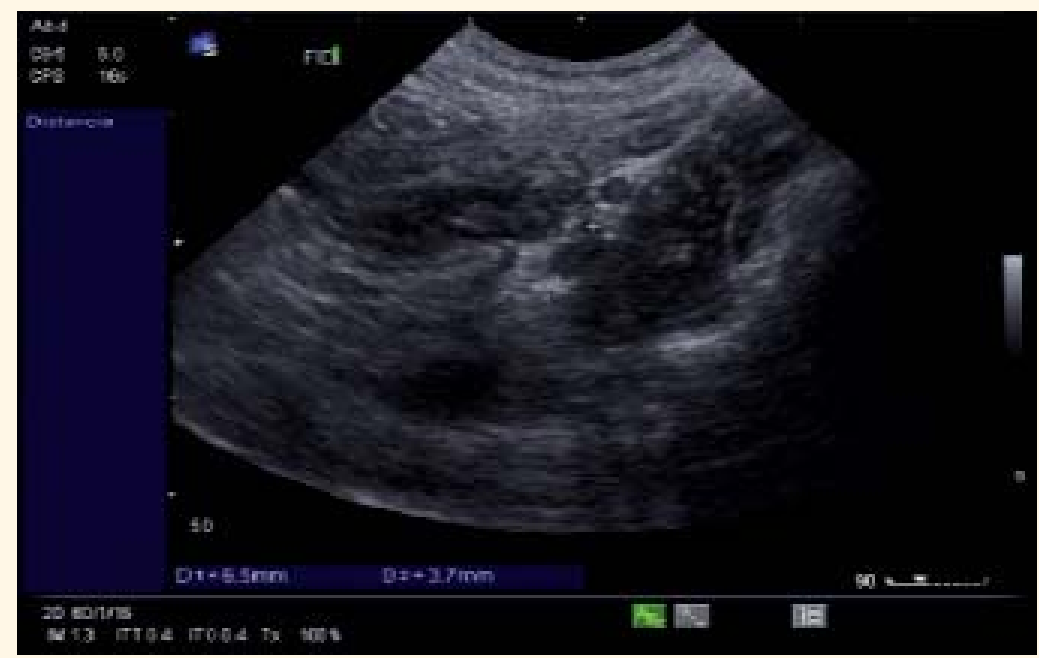

Figura 2. Imagen ecográfica de invaginación intestinal sin estudio doppler.

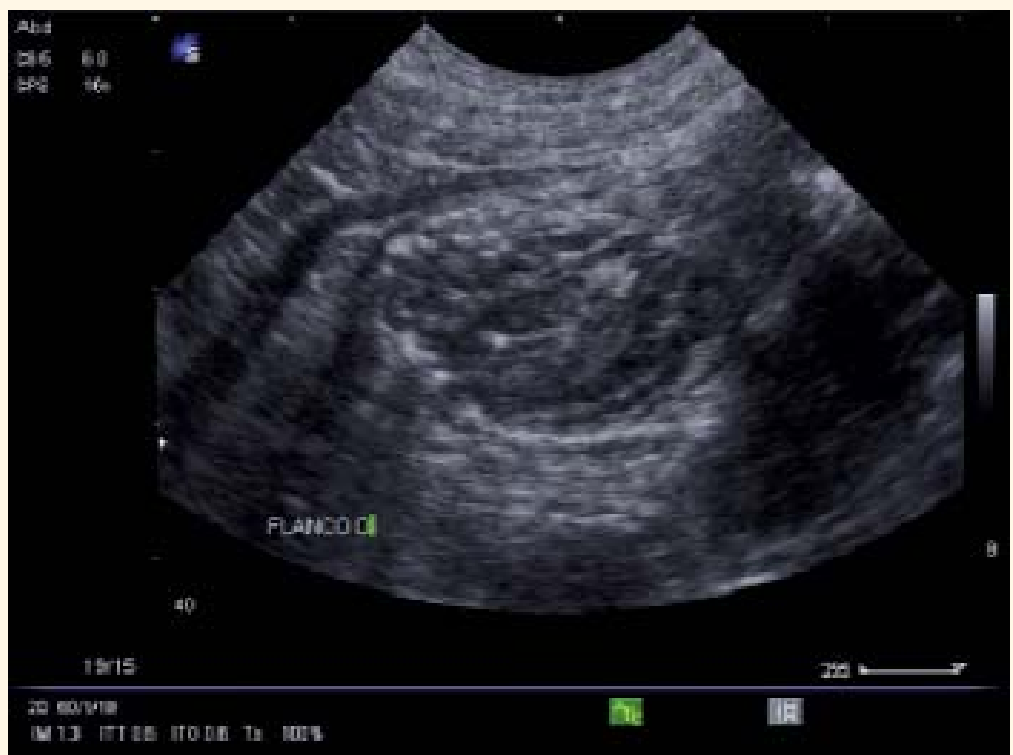


Figura 3. Imagen ecográfica de invaginación intestinal con estudio doppler.

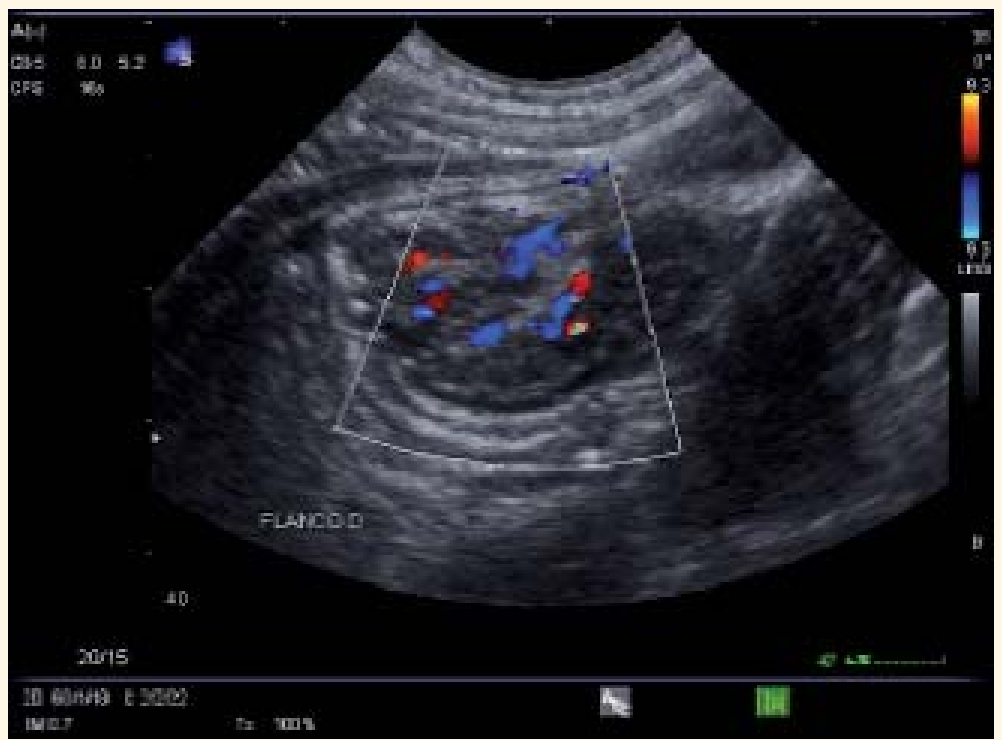

\section{Discusión}

La invaginación intestinal se considera una de las causas más frecuentes de obstrucción intestinal en el grupo de edad comprendido entre los 3 meses y los 6 años de edad. Debe sospecharse esta entidad en todo niño previamente sano que desarrolle crisis de dolor abdominal intermitente y hematoquecia. Otros síntomas clásicos asociados son los vómitos o la palpación de una masa abdominal más o menos alargada. La presencia estereotipada de estos síntomas permite al pediatra el diagnóstico rápido y su tratamiento de manera precoz, mejorando en estos casos las cifras de morbimortalidad asociadas a esta patología. Sin embargo, el dolor abdominal puede estar ausente o enmascarado por alteraciones neurológicas tales como apatía, somnolencia, hipotonía generalizada, escasa reactividad a estímulos dolorosos, nerviosismo, anorexia sin vómitos asociados e incluso convulsiones. Puede ser frecuente que el nivel de conciencia del paciente fluctúe $y$, en algunos casos, puede ser indistinguible del cuadro neurológico del paciente séptico'.

Ante una alteración del nivel de conciencia debemos plantearnos las causas potenciales de mayor o menor gravedad incluyendo: causas infecciosas (meningi- 
tis, encefalitis, sepsis), tumorales, que afecten al sistema nervioso central y tóxico-metabólicas. Dentro de este diagnóstico diferencial, la patología abdominal muchas veces no es tenida en cuenta, lo cual se traduce en la aplicación de técnicas diagnósticas invasivas innecesarias (TC de estructuras centrales, punción lumbar) y en un retraso del diagnóstico y el tratamiento, con el empeoramiento pronóstico que ello implica².

La etiología de la invaginación intestinal es desconocida en un 80-90\% de los casos. Se postula que un probable origen de esta patología se encontraría en una inflamación que acontece en las placas de Peyer y que actuaría como estímulo del peristaltismo intestinal para intentar expulsar la masa, produciendo la invaginación. Basándose en esta teoría, en algunos casos se han observado puntos que actúan de guía de la invaginación, tales como muñones apendiculares, pólipos, adenopatías mesentéricas (bastante frecuentes en el transcurso de las infecciones virales gastrointestinales) ${ }^{2}$ e incluso, aunque de manera extraordinaria, hematomas intramurales, como los presentes en algunos casos de púrpura Schönlein-Henoch ${ }^{3}$.

La patogenia de la invaginación intestinal consiste en la introducción de una porción del tubo digestivo dentro de un segmento posterior a éste. El sangrado se produce por compresión mesentérica, que dificulta el retorno venoso, lo cual favorece la congestión y la edematización de la porción invaginada. La mucosa se torna hemorrágica y produce heces sanguinolentas, con o sin moco, denominadas heces en jalea de grosella.

Se han ofrecido muchas teorías para intentar explicar la aparición de alteraciones neurológicas en el contexto de una invaginación. La pérdida de fluidos y el desequilibrio electrolítico consecuente a los vómitos y al grado de obstrucción intestinal pueden ser algunas de las causas'. También se postula acerca de citocinas todavía no caracterizadas que estuvieran presentes en la pared intestinal y se liberaran ante la afectación isquémica de ésta. Otros señalan la existencia de ciertos opioides endógenos que pudieran ser responsables de la obnubilación y el enmascaramiento del dolor abdominal. Por último, sí que es bien conocido el papel de ciertas toxinas bacterianas capaces de inducir una encefalopatía, pero su función dentro de la invaginación intestinal no está todavía del todo clara ${ }^{4-7}$.

En un paciente hemodinámicamente estable con alteración del estado mental, la posibilidad de una patología intracraneal potencialmente grave debe ser la 
primera causa que descartar $y$, por tanto, nuestra prioridad diagnóstica.

De forma clásica, se considera que el gold standard para el diagnóstico es el enema opaco, si bien la ecografía abdominal ha desplazado a éste por ser menos invasiva ${ }^{2}$.

La resolución del cuadro se realiza mediante el uso de enemas de bario o aire 0 , como en nuestro caso, de manera espontánea. Las invaginaciones de evolución más prolongada, o aquellas que se acompañan de afectación hemodinámi$\mathrm{ca}$, requieren cirugía. El estado neurológico del niño retorna a la normalidad al instante o unas horas después de que la circulación sanguínea vuelva a estar res- tituida tras la reducción de la invaginación; este hecho se considera un factor predictor de éxito ${ }^{1}$.

Podemos concluir que la invaginación intestinal puede cursar con sintomatología neurológica en la población pediátri$\mathrm{ca}$, por lo que ante inexplicables alteraciones del nivel de conciencia sin signos de focalidad neurológica, debemos considerar los cuadros abdominales dentro de nuestro diagnóstico diferencial. La ecografía es una técnica diagnóstica poco invasiva que puede ayudar en el diagnóstico diferencial de cuadros de abdomen agudo con sintomatología neurológica inexplicada en niños pequeños.

\section{Bibliografía}

1. Pumberger W, Dinhobl I, Dremsek P. Altered consciousness and lethargy from compromised intestinal blood flow in children. Am J Emerg Med. 2004;22:307-9.

2. Birkhahn R, Fiorini M, Gaeta TJ. Painless intussusception and altered mental status. Am J Emerg Med. 1999;17:345-7.

3. Katz S, Borst M, Seekri I, Grosfeld JL. Surgical evaluation of Henoch-Schonlein purpura. Experience with 110 children. Arch Surg. 1991;126: 849-53.
4. Shaoul R, Gazit A, Weller B, Berman S, Jaffe M. Neurological manifestations of an acute abdomen in children. Pediatr Emerg Care. 2005;21: 594-7.

5. D'Escrienne MM, Velin P, Filippigh P, Steyaert $\mathrm{H}$, Valla JS, Perelman S, et al. Lethargic form of acute intestinal intussusception in an infant. Arch Pediatr. 1996;3:44-6.

6. Sangermani R, Gibelli M, Vaglia P, Vaccari R. Changes in the consciousness state as a symptom of intestinal invagination onset. Pediatr Med Chir. 1992;14:623-5.

7. Tenenbein $M$, Wiseman NE. Early coma in intussusception: endogenous opioid induced? Pediatr Emerg Care. 1987;3:22-3. 